\title{
FreeDOM: A Transferable Neural Architecture for Structured Information Extraction on Web Documents
}

\author{
Bill Yuchen Lin* \\ University of Southern California \\ Los Angeles, California, USA \\ yuchen.lin@usc.edu \\ Nguyen Vo \\ Google \\ Mountain View, California, USA \\ nguyenvo@google.com
}

\author{
Ying Sheng \\ Google \\ Mountain View, California, USA \\ yingsheng@google.com \\ Sandeep Tata \\ Google \\ Mountain View, California, USA \\ tata@google.com
}

\begin{abstract}
Extracting structured data from HTML documents is a long-studied problem with a broad range of applications like augmenting knowledge bases, supporting faceted search, and providing domain-specific experiences for key verticals like shopping and movies. Previous approaches have either required a small number of examples for each target site or relied on carefully handcrafted heuristics built over visual renderings of websites. In this paper, we present a novel two-stage neural approach, named FrEEDOM, which overcomes both these limitations. The first stage learns a representation for each DOM node in the page by combining both the text and markup information. The second stage captures longer range distance and semantic relatedness using a relational neural network. By combining these stages, FrEEDOM is able to generalize to unseen sites after training on a small number of seed sites from that vertical without requiring expensive hand-crafted features over visual renderings of the page. Through experiments on a public dataset with 8 different verticals, we show that FrEEDOM beats the previous state of the art by nearly $3.7 \mathrm{~F} 1$ points on average without requiring features over rendered pages or expensive hand-crafted features.
\end{abstract}

\section{CCS CONCEPTS}

- Information systems $\rightarrow$ Web mining; Data extraction and integration.

\section{KEYWORDS}

structured data extraction, web information extraction

\section{ACM Reference Format:}

Bill Yuchen Lin, Ying Sheng, Nguyen Vo, and Sandeep Tata. 2020. FreeDOM: A Transferable Neural Architecture for Structured Information Extraction on Web Documents. In Proceedings of the 26th ACM SIGKDD Conference on Knowledge Discovery and Data Mining (KDD '20), August 23-27, 2020, Virtual Event, CA, USA. ACM, New York, NY, USA, 11 pages. https://doi.org/ $10.1145 / 3394486.3403153$

${ }^{*}$ The work was done while BYL was a research intern at Google AI.

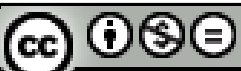

This work is licensed under a Creative Commons Attribution-NonCommercialNoDerivs International 4.0 License.

KDD '20, August 23-27, 2020, Virtual Event, CA, USA

(c) 2020 Copyright held by the owner/author(s).

ACM ISBN 978-1-4503-7998-4/20/08

https://doi.org/10.1145/3394486.3403153

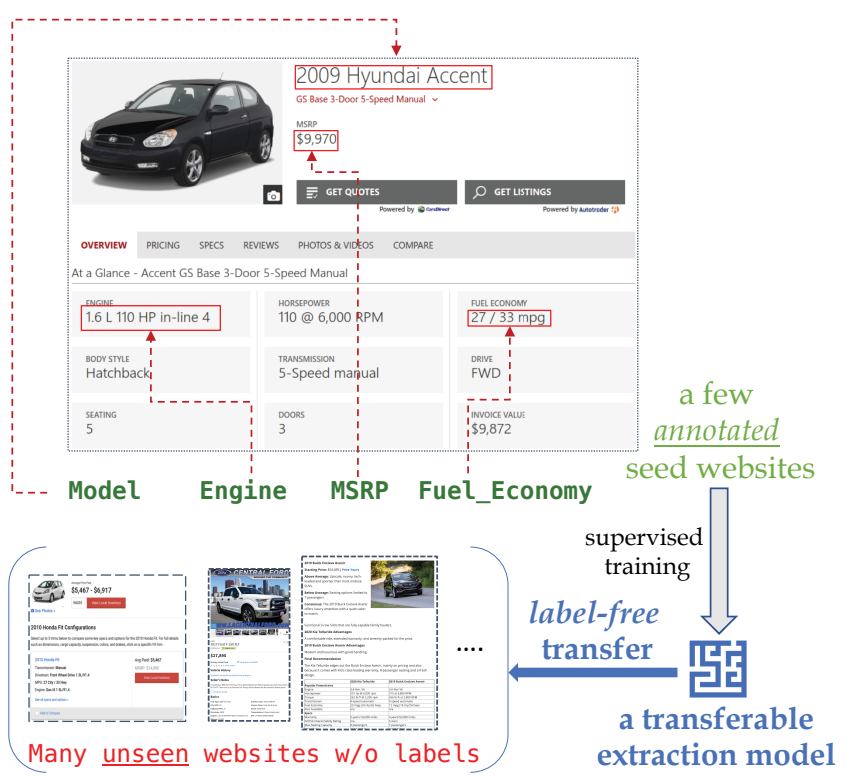

Figure 1: Learning a transferable extraction model that supports label-free adaption on unseen websites.

\section{INTRODUCTION}

Extracting structured information from web-pages is critical for large-scale knowledge base construction [3, 8, 9, 42]. This in turn has various downstream applications such as knowledge-aware question answering [6,23,39], recommendation systems [27, 28, 41], temporal event reasoning [33, 40], etc. Most recent advances in information extraction focus on neural models for extracting entities and relations on plain texts (i.e. natural language sentences) [35, 46]. However, these neural information extraction methods do not work well on web documents which are richly laid out and contain both natural language and markup information.

In this paper, we focus on extracting structured data with given attributes from detail pages. A detail page describes a single entity such as an IMDB page of a particular movie or an Amazon page about a certain product. For instance, consider that we want to extract information about vehicles from various websites and are interested in four attributes: \{model, MSRP, engine, fuel-economy\}. Conventional web data extraction methods are based on "wrapper 
induction" [17]. For each website, they require a handful of humanannotated pages and derive a pattern-based program that can work on other pages within the same website. This approach yields very high accuracy (over 95\% [13]). However, these wrapper-based methods need human annotations for each new website. This makes them expensive, time-consuming, and thus less practical when we have a large number of target websites of interest. In this paper, we are interested in an approach where we can learn from only a few annotated seed websites but then generalize to many unseen sites without additional labels (Figure 1).

Prior work primarily focused on exploiting visual patterns using carefully crafted features [14] . These rendering-based methods have two major drawbacks: 1) they are expensive since they require downloading all external files including CSS, javascript, and images to render the page to compute visual features; 2) they require carefully crafted heuristics around visual proximity to work well with these expensive features. In this paper, we propose a novel twostage neural architecture, named FREEDOM, that can be trained on a small number of seed websites and generalize well to unseen websites without requiring any hand-engineered visual features.

The first stage of our framework (Sec. 3) learns a representation for each node in the DOM tree ${ }^{1}$ of the page and classifies it into one of the target fields. This node-level module combines neighboring character sequences, token sequences, as well as markup (HTML elements) to learn a combined representation for the node. We propose a combination of CNNs and LSTMs and show that it can effectively encode useful features in DOM nodes.

These node representations are encoded individually and inevitably lose some global information useful for an extraction task. In particular, only relying on local node features can cause failure when value nodes have no obvious patterns themselves or their local features are very similar to other non-value nodes. To mimic the signal that may be available through visual features used in rendering-based methods, we use a relational neural network as our second module (Sec. 4). This allows us to model the relationship between a pair of elements using both distance-based and semantic features. The rationale behind this is to learn more global representations of node pairs so that we can jointly predict node labels instead of relying only on local features.

Extensive experimental results on a large-scale public dataset, the Structured Web Data Extraction (SWDE) corpus [14], show that our model consistently outperforms competitive baseline methods by a large margin. The proposed FrEEDOM is able to generalize to unseen sites after training on a small number of seed sites. In fact, we show that with training data from just three seed sites, our approach out-performs techniques that use explicit visual rendering features by $3.7 \mathrm{~F} 1$ points on average. To the best of our knowledge, our framework is among the first neural architectures that efficiently obtains high-quality representations of web documents for structured information extraction.

\section{PROBLEM OVERVIEW}

In this section, we describe the extraction problem formally before outlining the solution architecture.

\footnotetext{
${ }^{1}$ A DOM Tree, associated with a web page, is a collection of nodes, where each node has its address named XPath and original HTML content.
}
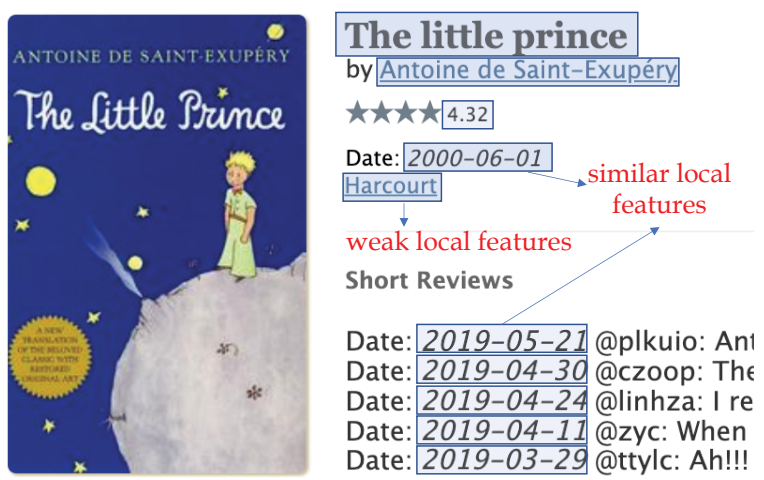

Figure 2: The two reasons why we need to model structured dependency via relational features. 1) The node "Harcourt" should be predicted as Publisher, but was classified as None by node-level classifier because it does not have very strong local features. 2) The top PublishDate values "2000-06-01" and the bottom review dates "2019-xx-xx" share very similar local features, and thus the node-level model tends to predict all as None because the majority have None labels.

\subsection{Problem Formulation}

Given a vertical $v$ (e.g. Auto) and a set of websites $W_{v}$ in this vertical, we assume that only a few seed websites $W_{v}^{s}$ are labeled by human annotators, and the other sites are unseen and unlabeled target websites $W_{v}^{t}$. A website $w_{i}$ can be viewed as a collection of web pages $w_{i}=\left\{p_{j}\right\}$ that share similar layout structures. Each page $p_{j}$ describes a single topic entity (e.g. a car model in Auto vertical) and is parsed to a DOM tree $t_{j}$.

We formulate the extraction task as node tagging on DOM Trees. For instance, the HTML page in Figure 1 can be parsed into a DOM tree, and each element on this page that we can view in the browser corresponds to a unique XPath and a DOM node. Structured information extraction can be viewed as classifying each node into one of the pre-defined vertical-specific groups: "Model", "Engine", "MSRP", "Fuel_Economy", and None. Note that we only need to tag leaf nodes that have textual content. Following the convention in structured web information extraction [14], we assume a single leaf node can correspond to at most one target field.

The research question now is to learn a model $\mathcal{M}$ that is trained with labeled pages in a few seed sites $W_{v}^{s}$, but still works well on unseen target sites $W_{v}^{t}$ in the same vertical $v$, even though their templates may be completely different. In other words, we want to learn site-invariant feature representations such that the model generalizes to unseen sites without additional human effort.

\subsection{Model Architecture}

We solve this problem using a two-stage architecture. The first stage learns a dense representation for each DOM node by combining both markup and textual content from its neighborhood. We find that using both markup and text can result in better embeddings. This dense representation is used to train a classifier to predict if the node corresponds to a target field or is None.

We find that directly using the outputs from the first stage (i.e. node-level classifier) has a common failure: the model fails to find 


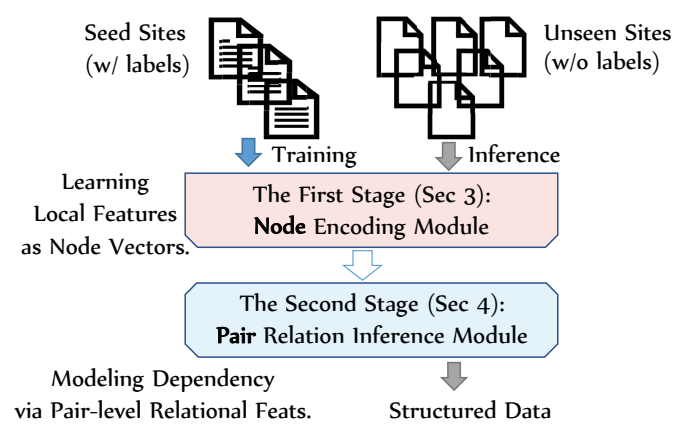

Figure 3: The overall workflow of FREEDOM .

some target fields and ends up labeling them None. For these fields, the ground truth node either often shares very similar local features as other None nodes or has very weak local signal (e.g. lacking of important anchor words). See the examples of Publisher and PublishDate in Fig. 2.

In retrospect, these problems were not surprising since the node representations captures local information rather than long-range patterns and constraints in the DOM tree. Both cases are easy for humans to recognize because we can infer by their relations with other fields such as Author and Title. In order to overcome these problems, we use a second stage node-pair model to incorporate information from parts of the document that are farther away. The core idea was inspired by the relation network module [36] for modeling pairs of spots in images. The node pair model is trained on pairs of DOM nodes and predicts if each of the nodes in the pair contains some value. The predictions of the second stage allows us to carefully discard low-confidence predictions from the first stage.

One question that immediately comes to mind is why not build on a sequence labeling model like BiLSTM-CRF [19] that is known to perform well on structured prediction tasks. In Section 5.3, we tackled this question and found that sequence tagging models built on top of the node embeddings produced by the first stage work slightly worse than simply using the node-level classifier. However, the pair-level module is able to better encode long-range patterns and outperforms these alternatives. See Section 5 for experiments and analysis comparing with a BiLSTM-CRF.

\section{NODE ENCODING MODULE}

We describe the first stage of the FREEDOM architecture which solves a node-level classification task for each "variable"2 leaf node in a DOM tree. We learn a dense representation for each node by combining several features associated with each node.

\subsection{Input Features}

We consider three primary sources of information to learn a representation for each node: 1) node text (i.e., text tokens in the node), 2) preceding text, and 3) discrete features based on markup and type annotations. As shown in Figure 4, the node-level module first encodes each of these node-level features individually with

\footnotetext{
${ }^{2}$ Variable nodes (with the same XPath) have different contents across different pages (as shown in blue boxes in Figure 2). Thus, we can ignore nodes that are common boilerplate, such as navigation bars, headers, footers, etc. See A.1 for more details.
}

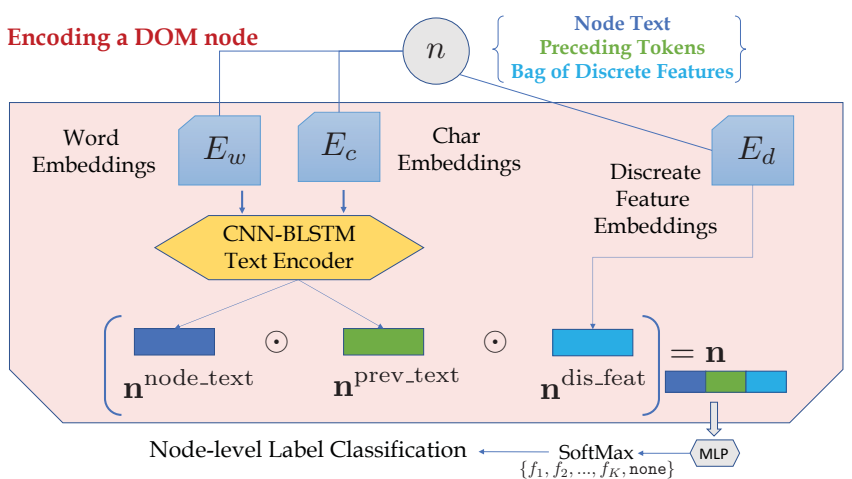

Figure 4: Learning a node-level classifier with trainable comprehensive node embeddings from three different views.

separate networks. These embeddings are concatenated and we train a simple feed-forward network over this representation via optimizing the SoftMax classification loss.

Node text embeddings. The text inside a leaf DOM node $n$ can be seen as a sequence of tokens $\left\{w_{i}\right\}$. Each token itself is a sequence of characters $\left\{c_{j}\right\}_{i}$, where $w_{i} \in \mathcal{W}$ and $c_{j} \in C$. We use a twolevel neural sequence encoder to capture both sub-word and wordlevel semantics. Modeling char-level semantics is beneficial for modeling the morphological patterns in values of different fields. General patterns consisting of out-of-vocabulary words and special characters can be learned with char-level CNNs and word-level LSTMs without explicit expert knowledge [14]. For example, a node for the "Fuel_Economy" field in Auto vertical could be "city 33 hwy 27 ", and capturing such "city $x x$ hwy $y y$ " patterns requires both token-level and char-level representations.

Similar to the architecture used for named entity recognition [29], we first initialize a character embedding look-up table $\mathbf{E}_{c} \in \mathbb{R}^{|C| \times \operatorname{dim}_{c}}$ for all characters $(C)$ where $\operatorname{dim}_{c}$ is a hyper-parameter for the dimension of character embedding vectors. Then, we use a convolutional neural network $(\mathrm{CNN})$ to efficiently encode the sequences of character embedding vectors. After pooling by the $\mathrm{CNN}$, we have a character-level word representation, which is further concatenated with a word-level vector initialized using external StanfordGloVE [32] word embeddings. On the top of such concatenated word representations, we employ a bidirectional LSTM network for contextually encoding the whole sequence forward and backward. The final node text representations $\mathbf{n}^{\text {node_text }}$ can be derived as follows:

$$
\begin{gathered}
\mathbf{c}^{\mathbf{i}}=\operatorname{CNN}\left(\left\{c_{1}, c_{2}, \ldots, c_{|w|}\right\}\right) ; \mathbf{t}_{\mathbf{i}}=\left[\mathbf{w}^{\mathbf{i}} \odot \mathbf{c}^{\mathbf{i}}\right] \\
\mathbf{n}^{\text {node_text }}=\operatorname{AVG}\left[\operatorname{LSTM}_{f}\left(\left\{\mathbf{t}_{1}, \mathbf{t}_{2}, \ldots\right\}\right) \odot \operatorname{LSTM}_{b}\left(\left\{\mathbf{t}_{|\mathbf{n}|}, \mathbf{t}_{|\mathbf{n}|-1}, \ldots\right\}\right)\right]
\end{gathered}
$$

where $[\cdot \odot \cdot]$ denotes concatenation.

Preceding text embeddings. The text in the nodes that precede the target node is clearly a valuable signal. Suppose that we are extracting the "MSRP" nodes on a page, and the ground truth is the node with text " $\$ 9,970$ ". There may be many other nodes in the page also containing similar texts (e.g. a node with " $\$ 9,872$ ') denoting the invoice price instead of the target MSRP field. The text 
preceding the target node, with tokens like "MSRP :" and "Invoice Price :" in our example, is clearly a critical signal.

We use the same text-encoding module (i.e. the CNN-BLSTM network) to encode the content preceding the target node (i.e. the 10 previous tokens). This results in an additional vector representing the previous node text as $\mathbf{n}^{\text {prev_text }}$.

Discrete features. The third source of information comes from markup and type-specific annotations on the content in the target node. We model these as a bag of discrete features. The DOM leaf type of the leaf node such as '<h1>', '<div>', '<li>', '<span>' can be useful for identifying some fields. For instance, key fields like like model names and book titles are often encoded using $<$ h1 $>$. We also apply a set of straightforward string type checkers (e.g. those in the NLTK toolkit) to know if the node text contains any numbers, dates, zip codes, URL link, etc. Note that a single node can have multiple such discrete features of these two groups. We model these as follows: we first maintain a look-up embedding table

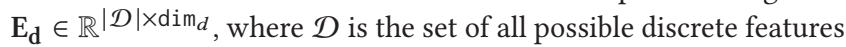
and $\operatorname{dim}_{d}$ is the dimension of feature vectors. We utilize max pooling to map two multi-hot discrete feature vectors respectively to each bag of features $\mathbf{d}_{1}, \mathbf{d}_{2} \in\{0,1\}^{|\mathcal{D}|}$ to real space vectors of dimension dim $_{d}: \mathbf{n}^{\text {dis_feat }}=\left[\mathbf{d}_{1} E_{\mathbf{d}} \odot \mathbf{d}_{2} \mathrm{E}_{\mathbf{d}}\right]$ by matrix multiplication. This can be seen as the concatenation of two max-pooled vectors over the associated feature-vectors in each bag of discrete features. The three sources of information described are each encoded into dense vectors. These three vectors are concatenated to provide a comprehensive representation of the node in the DOM tree.

\subsection{Learning Node Vectors via Classification}

The concatenated output representation is connected to a multilayer perceptons (MLPs) for multi-class classification via a SoftMax classifier (see Figure 4) that is illustrated in the following equations:

$$
\begin{gathered}
\mathbf{n}=\left[\mathbf{n}^{\text {node_text }} \odot \mathbf{n}^{\text {prev_text }} \odot \mathbf{n}^{\text {dis_feat }}\right] \\
\mathbf{h}=\operatorname{MLP}(\mathbf{n}), \text { where } \mathbf{h} \in \mathbb{R}^{K+1} ; l=\arg \max _{i} \frac{e^{\mathbf{h}_{i}}}{\sum_{j=1}^{K+1} e^{\mathbf{h}_{j}}}
\end{gathered}
$$

Recall that our goal is to assign a label $l$ for each node $n$ in any page $p$, where $l$ is either a pre-defined field (out of $K$ types) or a "none" label. Thus, the outputs from the SoftMax represent the distribution over the target labels, and the intermediate output over which the MLP is the learned combined representation for each node. The outputs from the first stage can directly be used as (tentative) labels for each node.

\section{RELATION INFERENCE MODULE}

As we previously mentioned, directly using the outputs from the first stage may end up not predicting a required field for any of the nodes in the page. Recall the example in Figure 2, the first stage model assigns a 'None' to the PublishDate field node since it has very similar features (e.g. "Date" as preceding text) as many 'None' nodes. Missing the prediction of the Publisher field is instead because its local features are not strong enough to generalize to unseen sites. These issues are expected given the first stage model does not use non-local information and does not take into account
Figure 5: Learning node-pair representations from different signals and the pair-level classification model.

global page-level constraints. Individually classifying node labels inherently limits the model's ability of using a broader context for jointly classifying labels of multiple nodes within a page. Instead, we argue that modeling conditional dependencies among the nodelevel neural features can help improve the output predictions.

Knowing that value nodes of related fields are generally presented in a similar layout across sites can be valuable in distinguishing between these similar nodes. Figure 9 in Appendix A shows that pairwise distances between target fields are correlated across sites. Rendering-based models usually design heuristic methods to use visual distance to deal with these problems in the post-processing stage, which are more expensive and need human knowledge. The success of visual distance features used in rendering based models also supports the claim that such relations between fields are strong transferable patterns [14].

How can we model such distance patterns in a neural network for learning end-to-end instead of hard-coding as rules? We propose to do this in the second stage of our architecture to capture conditional dependencies between nodes when predicting node labels. However, modeling the dependencies among a huge number of nodes in a DOM tree structure is challenging. Inspired by the recently proposed relation network module [36] for modeling pairs of spots in images, we propose to directly model the relation features of node-pairs on DOM trees. This should allow us to efficiently encode relational features between them. With such relational features, the conditional dependencies can be used to produce more precise structured predictions.

We first propose a way to construct node pairs (Sec. 4.1) based on the first-stage outcomes, then we learn a node-pair relation network (Sec. 4.2) for encoding node-pairs with additional distancebased features, and finally we show how to aggregate the nodepair predictions to improve the predictions from the first stage for structured data extraction (Sec. 4.3).

\subsection{Node-Pair construction}

A typical web page has over 300 nodes (see Tab. 1) that our model should predict labels for. Considering that the full combinations (about $300^{2}$ node-pairs) is huge, we instead only consider the following subsets to reduce the computational cost in the second stage. Based on the first-stage outputs, we use the raw output scores $\left(\mathbf{h}_{\mathbf{i}}\right)$ to filter the nodes as follows. First, we divide the fields to two groups: 
certain fields and uncertain fields depending on whether there is at least one node predicted for it. For the certain fields, we take each of the nodes predicted by the first-stage module as the anchor nodes for the field. While for uncertain fields, we instead take the top $m$ nodes with the highest score for that field to construct nodepairs. Say we have $K$ fields in total, and $T$ of them are certain fields, then we have $T *(T-1)+2 * T *(K-T) * m+(K-T) *(K-T-1) * m^{2}$ node-pairs ${ }^{3}$ to score. For each node-pair, we assign a pair-label according to whether head/tail node is a value/none. That is, we use the label space as $\{(\mathrm{N}, \mathrm{N}),(\mathrm{N}, \mathrm{V}),(\mathrm{V}, \mathrm{N}),(\mathrm{V}, \mathrm{V})\}$, where $\mathrm{N}=$ "None" and $V=$ "Value". Thus, the learning objective of the second-stage is to predict the pair-labels $\left(l_{\text {head }}, l_{\text {tail }}\right)$ of each node-pair $\left(n_{\text {head }}, n_{\text {tail }}\right)$. The key rationale behind our pair construction process is that we hope the certain fields can work as pivots for the model to extract confusing fields by their distance-based features.

\subsection{Node-pair relation networks}

We propose two ways of modeling distances between nodes: XPath sequences and positional embeddings. We combine both to learn an encoding for the node-pair distance and concatenate it with the first-stage output (i.e. node vectors) as the representation of node-pairs for learning. Denoting the head and tail nodes as $n_{\mathrm{h}}$ and $n_{\mathrm{t}}$ respectively, we summarize the three views of node-pair representations in Figure 5).

An XPath of a DOM node can be seen as a sequence of HTML tags like ["<html>", "<body>", "< div>", "< $<$ l>", "<li>"]. Nodes that are closer in the rendered result by web browsers usually have also similar XPath sequences. The distance in their XPaths can thus be used as a proxy for estimating their distances in rendered pages by web browsers. However, the underlying distance between a pair of XPaths is hard to model directly for generalizing to unseen websites. Inspired by neural sequence modeling, if we could represent each $\mathrm{XPath}$ as a vector and then their hidden differences can be further modeled by the non-linearity of neural networks. Specifically, we train a bi-directional LSTM (BiLSTM) to get the representation of an XPath sequence, such that the vectors of all tags in it is encoded as a dense vector. We denote the XPath LSTM output vector of head and tail node as $\operatorname{xpath}\left(n_{h}\right)$ and $\operatorname{xpath}\left(n_{t}\right)$ respectively.

The position of a node in the page is also an explicit signal to describe the distance. Thus, we further utilize positional embeddings for encoding, which is commonly used in relation classification tasks [44]. We take the positions of each node in a limited range of integers between 0 and $L$ (say $L=100$ ). Then, we will have an embedding matrix $E_{\text {pos }}$ to learn, and thus we have $\operatorname{pos}\left(n_{h}\right)$ and $\operatorname{pos}\left(n_{t}\right)$ for positional features of the two nodes.

From the first-stage model-level predictions, each node has been represented as a node vector $\mathbf{n}$. We continue using such learned representation of all nodes, denoted as $\mathbf{n}_{\mathrm{h}}$ and $\mathbf{n}_{\mathrm{t}}$, which respectively represent the local features of the two nodes by the first-stage model. Finally, we concatenate all the three views of relational node-pair features together and have:

$$
\mathbf{r}=\left[\mathbf{n}_{\mathrm{h}} \odot \mathbf{n}_{\mathrm{t}} \odot \operatorname{xpath}\left(n_{h}\right) \odot \operatorname{xpath}\left(n_{t}\right) \odot \operatorname{pos}\left(n_{h}\right) \odot \operatorname{pos}\left(n_{t}\right)\right]
$$

\footnotetext{
${ }^{3}$ Note that $2 * T *(K-T)$ stands for the number of field pairs containing one certain and one uncertain fields and $* m$ means that we iterate the top $m$ nodes in uncertain fields to construct pairs. Similarly, $(K-T) *(K-T-1)$ is the number of pairs where both fields are uncertain. $T *(T-1)$ is the number of certain fields pairs.
}

On top of this, we further build a multi-layer feed-forward neural networks as a SoftMax multi-class classifier. Unlike what we do in the node-level module, here our target label space for node-pairs are \{none-none, none-value, value-none, value-value\}.

\subsection{Label-pair aggregation and voting.}

Pair-labels aggregation. Recall that in the pair construction stage (Sec. 4.1), we choose one node for each certain field (out of $T$ ) and $m$ nodes for each uncertain field (out of $K-T$ ). Thus, each node in the node-pairs is already assigned a field-type. We only need to re-consider candidate nodes for uncertain fields and determine if they should be treated as a Value or a None for the target field. The prediction of the second-stage model for a node pair assigns a Value or a None to nodes in the pair. A node involved in $X$ pairs then gets $X$ labels. It is considered as Value if $>=N$ labels among the $X$ labels are Value, where $N$ is a hyper-parameter. This is the output from the second stage.

Site-level XPath Voting. Inspired by prior works on web content redundancy $[12,20]$, we argue that many value nodes of a field tends to come from the same XPath across different pages in the same site. Thus we find the XPath selected as the field value by the majority pages and correct the rest of the pages (if there is inconsistent outliers) to extract field value from this XPath as well.

\section{EXPERIMENTS}

The experiments in the section are designed to answer two broad questions. First, how well does FreeDom generalize to unseen sites in a given vertical after being trained on examples from a small number of seed sites? We present a comparison with previous methods proposed in the literature. We show that FreeDom can generalize better to unseen sites after training on just three seed sites and beats the previous SOTA by 3.7 points without using visual renderings. Second, how much do various design choices contribute to our accuracy? We present an ablation study including a comparison to sequence labeling baselines (e.g. BLSTM-CRF) showing the contributions of the two modules in the solution.

\subsection{Dataset and Set-up}

We use the publicly accessible Structured Web Data Extraction (SWDE) dataset [14] for all our evaluation tasks. As shown in Tab. 1, the SWDE dataset has 8 verticals and 10 websites for each vertical. Each vertical specifies $3 \sim 5$ fields to extract. Each website has hundreds of pages, where a page has about 300 variable nodes for us to classify. We use open-source LXML library ${ }^{4}$ for efficiently processing each page to get its DOM tree structure.

Experimental settings. We randomly select $k$ seed websites as the training data to train different extraction models, and test the models on the pages in other remaining $10-k$ sites (i.e. the target sites) where no label at all is used for transferring trained models. For each vertical, we take 10 cyclic permutations after fixing an order within the websites. Obviously, the same website is never present in training and test data in any given experiment. This setting is close to the real application scenarios we care about

$\overline{{ }^{4} \text { https://lxml.de/ }}$ (Other alternatives can also work.) 


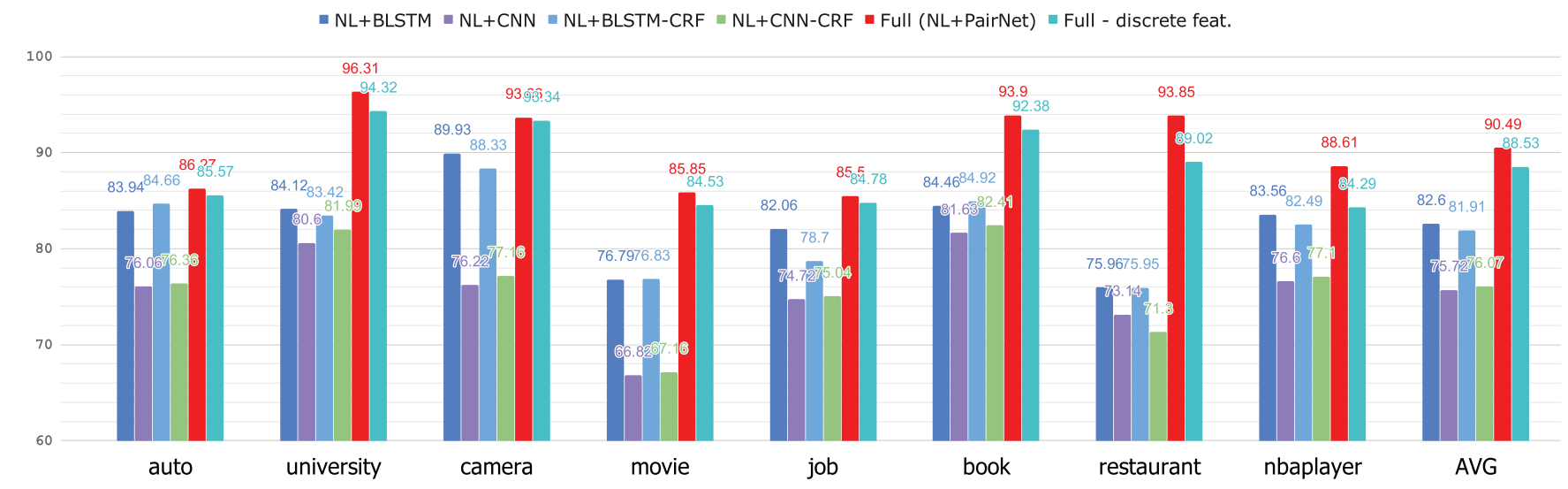

Figure 6: Ablation study results by replacing the proposed Pair Network with other node-sequence labeling-based models (CNN/BLSTM + an optional CRF layer) when using three seed websites in each vertical $(k=3)$. We also show the effectiveness of Freedom-Full if discrete features are disabled (w/o discrete feat.).

\begin{tabular}{ccccc}
\hline Vertical & \#Sites & \#Pages & \#Var. Nodes & Fields \\
\hline Auto & 10 & 17,923 & 130.1 & model, price, engine, fuel_economy \\
Book & 10 & 20,000 & 476.8 & title, author, isbn, pub, date \\
Camera & 10 & 5,258 & 351.8 & model, price, manufacturer \\
Job & 10 & 20,000 & 374.7 & title, company, location, date_posted \\
Movie & 10 & 20,000 & 284.6 & title, director, genre, mpaa_rating \\
NBA Player & 10 & 4,405 & 321.5 & name, team, height, weight \\
Restaurant & 10 & 20,000 & 267.4 & name, address, phone, cuisine \\
University & 10 & 16,705 & 186.2 & name, phone, website, type \\
\hline
\end{tabular}

Table 1: The statistics of the public SWDE dataset.

where training data is generated from a small number of seed sites, and we want to learn to extract for dozens of other unseen sites where we may have no labels. We use the same evaluation metrics following the authors of SWDE [14] and compute the page-level F1-scores in each vertical and field. Note that the authors of SWDE do not consider the cases where there are multiple values for the same field in a page and thus we take our top-1 predictions.

\subsection{Baseline methods and FreeDOM variants}

The Stacked Skews Model (SSM) [2] utilizes a set of human-crafted features for aligning the target unseen website DOM trees to the pages in existing annotated seed websites with tree alignment algorithms [45] that are time-consuming. The authors of the SWDE dataset applied this method under the same problem setting and stated that this was the state-of-the-art method that did not require visual rendering features. Thus, this model is the closest to ours in terms of assumptions on available supervision.

Hao et al. [14] propose a set of rendering-feature baseline methods that use the visual features to explore the distance between each block in the web browser rendered result. The visual distances have proven to be very strong features for structured data extraction in web documents. Note that the rendering requires downloading and executing a large amount of external scripts, style files, and images, which are extremely time/space-consuming. Further, their approach requires a set of pre-defined, human-crafted patterns for recognizing values of fields (e.g. price, length, weight) using prefix and suffix n-grams.
This baseline method has three variants, which we named as Render-PL, Render-IP, and Render-Full respectively. Hao et al. [14] first use the visual-distance features and human-crafted rules to learn a model to classify each node individually at the pagelevel (Render-PL). Using site-level voting like we mentioned in Sec. 4.3, they further improve the method by controlling inter-page consistency (Render-IP). Finally, they further propose a complicated heuristic algorithm for computing visual distances between predicted value nodes and adjust the predictions in a whole site ("Render-Full"). As we can see, these methods ${ }^{5}$ are highly dependent on the visual rendering to obtain strong distance features as well as hand-crafted patterns.

We also compare the proposed FreEDom model, named "FreeDOMFull", with the node-level model ("FreeDOM-NL"), which simply uses the output for the first stage. Comparing "FreeDOM-NL" and "FreeDOM-Full" can tell us the importance of our relation inference module (Sec. 4). We also adopt a series of recently proposed neural sequence modeling architectures, as the alternatives to the our proposed pair-level networks. These sequence labeling models are based on our node-level sequence encoder and an optional CRF [18] layer to perform node tagging. These are also reasonable baselines since they allow the model to learn from a longer inter-node dependency while performing structured predictions. According to the choices of the node sequence encoder and whether to impose CRF tagging layer, there are FreeDOM-NL+BLSTM, FreeDOM-NL+CNN, FreeDOM-NL+BLSTM-CRF, and FreeDOM-NL+CNN-CRF. Note that all of them are further improved with site-voting for fair comparisons. The tuned parameters of these models are reported in Appendix A.

\subsection{Experimental results and discussion.}

We first compare the overall performance of all baseline models and our full model using different numbers of seed websites. Then, we do a detailed ablation study on different verticals and fields to verify the effectiveness of our proposed two-stage framework.

\footnotetext{
${ }^{5}$ We report the numbers exactly from the paper of such models [14] without reimplementation since some of their resources are not publicly available or outdated.
} 

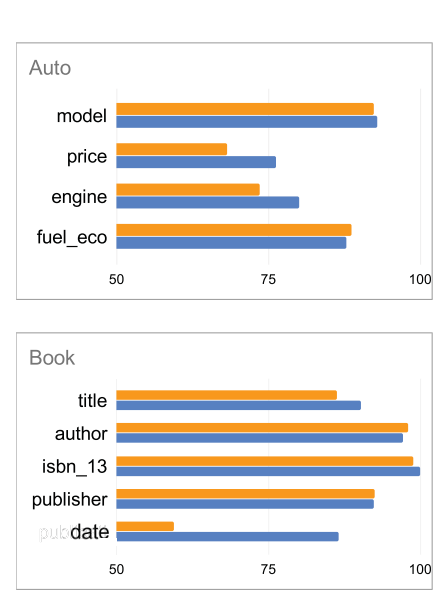
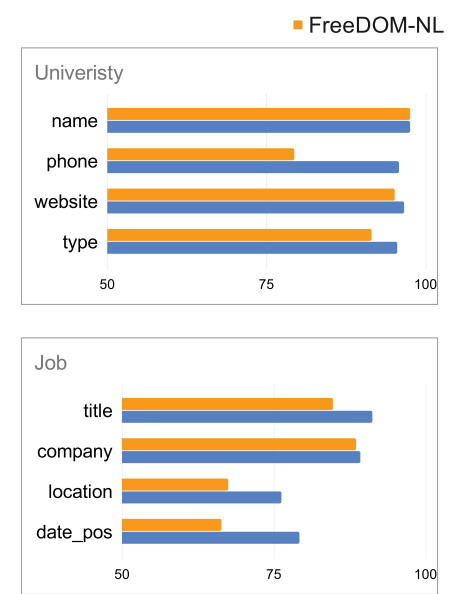
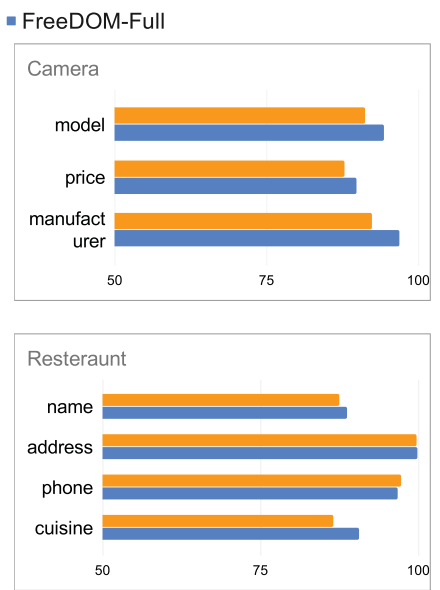
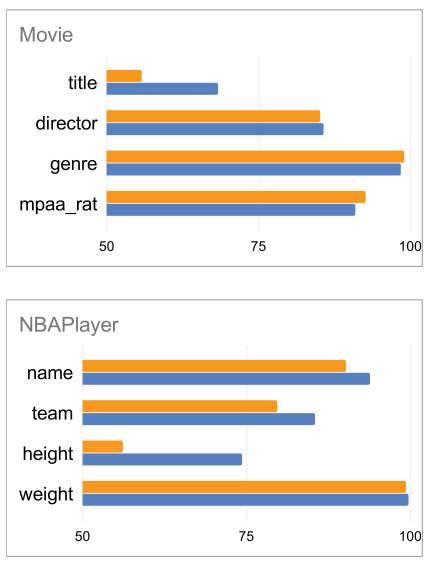

Figure 7: Per-field performance (F1\%) comparisons between FreeDOM-NL and FreeDOM-Full when using three seed websites.

\begin{tabular}{cccccc}
\hline Model $\backslash$ \#Seed Sites & $k=1$ & $k=2$ & $k=3$ & $k=4$ & $k=5$ \\
\hline SSM & 63.00 & 64.50 & 69.20 & 71.90 & 74.10 \\
Render-Full & $\mathbf{8 4 . 3 0}$ & 86.00 & 86.80 & 88.40 & 88.60 \\
FreeDOM-NL & 72.52 & 81.33 & 86.44 & 88.55 & 90.28 \\
FreeDOM-Full & 82.32 & $\mathbf{8 6 . 3 6}$ & $\mathbf{9 0 . 4 9}$ & $\mathbf{9 1 . 2 9}$ & $\mathbf{9 2 . 5 6}$ \\
\hline
\end{tabular}

Table 2: Comparing performance (F1-score) of the four typical methods including our FreeDOM using different numbers of seed sites (from 1 to 5). Each entry is the mean value on all 8 verticals and 10 permutations of seed websites, thus 80 experiments in total. Note that Render-X methods utilize rendering results that require huge amount of external resources than SSM and FreeDOM-X.

\subsubsection{Overall performance.}

As shown in Tab. 2, we extensively test the overall performance of four methods on how well they generalize to unseen sites using different numbers of seed sites for training. Note that each entry in this table is the mean of the F1 of 80 experiments: 8 verticals and 10 permutations of the $k$ seed websites. We can see that the full rendering-based model (Render-Full) perform the best when $k=1$, but when $k \geq 2$ the performance of our proposed full FreeDOM outperforms other methods by a large margin (nearly 4 absolute F1 increment over Render-Full and 18 over SSM when $k=5$ ).

As a side note ${ }^{6}$, the Render-PL's result is $70.63 \%$ and Render-IP's is $82.01 \%$ when $k=1$. It shows that rendering-based features and rule-based patterns can be stronger than our proposed neural architecture when there is very little training data. This is not surprising since these visual features were carefully crafted by humans to capture patterns present in SWDE. However, when using more than two seed websites, our neural architecture quickly matches the performance obtained from hand-crafted visual features. FreeDOM-NL achieves a similar performance with Render-Full (86.44 vs 86.80) when using 3 seed websites. With our novel pair-level networks (FreeDOM-Full) for learning relational features, the performance

${ }^{6}$ In their paper, only "Render-Full" has detailed results from $k=1$ to 5 and it is significantly better than the other two. Therefore, we mainly compare with Render-Full.

\begin{tabular}{cccccc}
\hline Vertical $\backslash$ \#Seed Sites & $k=1$ & $k=2$ & $k=3$ & $k=4$ & $k=5$ \\
\hline Auto & 84.78 & 83.33 & 86.27 & 88.37 & 88.50 \\
University & 89.18 & 92.73 & 96.31 & 94.90 & 97.88 \\
Camera & 86.85 & 89.24 & 93.66 & 94.52 & 95.01 \\
Movie & 77.35 & 82.43 & 85.85 & 87.60 & 88.87 \\
Job & 78.35 & 80.11 & 85.50 & 85.30 & 86.60 \\
Book & 87.25 & 92.45 & 93.90 & 95.45 & 95.47 \\
Restaurant & 81.07 & 88.54 & 93.85 & 92.89 & 94.67 \\
Nbaplayer & 73.71 & 82.03 & 88.61 & 91.26 & 93.46 \\
\hline AVG & $\mathbf{8 2 . 3 2}$ & $\mathbf{8 6 . 3 6}$ & $\mathbf{9 0 . 4 9}$ & $\mathbf{9 1 . 2 9}$ & $\mathbf{9 2 . 5 6}$ \\
\hline
\end{tabular}

Table 3: The performance (F1-score) of FreeDOM-Full of using different numbers of seed websites in different verticals.

is further improved. A detailed report about the performance of FreeDOM-Full on different verticals are shown in Table 3. We can see that the absolute improvement on F1 increases all the way to using 5 seed websites with gradually diminishing improvements.

\subsubsection{Ablation Study.}

To examine the effectiveness of our second-stage model, we replace the pair network with a set of popular neural sequence labeling models. These can be seen as alternative methods that can use global context for producing structured predictions. To ensure a fair comparison, we use the same first-stage model to compute the node representations. In the end, we also test if an additional layer can help decode tag sequences, which is commonly used in sequence tagging models [19, 21, 22]. As shown in Figure 6, we find that such sequence labeling-based models are all worse comparing to using the proposed pair networks in our second stage learning (even slightly worse than only using the node-level classifier). This is the case with both CNNs and BLSTM for encoding the sequences. Using CRF makes them even worse. This raises an interesting question: Why do neural sequence labeling models not work well with DOMTree node classification?

Recent research efforts show that The CNN/BLSTM-CRF models are powerful for encoding input sequences and decode them into tag sequences. However, we argue that sequences of DOM nodes ordered by their natural occurrence in web pages are not suitable for sequence learning models. The reasons are three-fold: 1 ) node 
sequences are usually too long, 2) structural dependencies are not sequential, and 3) the labels are too sparse. There are thousands of nodes in a DOM tree, and even if we filter all fixed nodes, the node sequences are still longer $(>100)$. Also, consecutive nodes in such sequences do not necessarily have label-wise relations. Such dependencies on labels usually exist in distant node pairs, such that CNN-based encoder loses a lot useful relational information. $\mathrm{CRF}$ is especially designed for capturing consistent label-transition probability, which is not evident in our sparse label sequences. Therefore, these sequence labeling models do poorly as alternatives, even with our node encoder.

Apart from that, we also show the importance of discrete features in our model (the last item in each group of Figure 6). We conclude that such discrete features are helpful while removing them would not harm the performance too much. Therefore, if running such discrete feature extractors might be a problem of users, they can still have a stronger model than using rendering-based methods.

5.3.3 Per-field Analysis. Figure 7 shows the detailed per-field performance comparison between FreeDOM-NL and FreeDOM-Full when $k=3$. We conclude that FreeDOM-NL can already perform very well on many fields, which means our proposed first-stage module learns great node-level representations. However, for certain fields that FreeDOM-NL is very bad like the publication_date in Book vertical, the FreeDOM-Full utilize the relational features to improve them very much, and in the end significantly improve the overall performance. Similarly, we find that FreeDOM-Full classifies better in the key fields (e.g. Title in Movie, and in fob). This is because such values usually do not have directly useful local features learned by node-level model, but they can be identified by our pair-level model with relational features and joint predictions.

\section{RELATED WORK}

Our work builds on research in wrapper induction in the data mining community and the contributions of neural models for information extraction tasks in the NLP community. We apply it in the context of modeling semi-structured web documents with neural networks. Specifically, we aim to build a more lightweight yet transferable model by getting rid of expensive rendering-based features and complex human-crafted algorithms.

Structured Data Extraction. Structured data extraction from web documents has been studied extensively in supervised settings $[1,10,37,38]$. Early works $[5,11,13,30]$ usually require a significant number of human-crafted rules or labels for inducing a wrapper (i.e. a program or model for extracting values of interested fields), which is only used for a particular web site. These wrappers are usually brittle when testing on unseen websites in the same vertical, although they can have high precision and recall on the training sites. Some recent works propose methods that can adapt to new websites. Zhai et al. [45] employed active learning methods that can find the most influential new examples in target websites for human annotators to label, and therefore adapting the existing wrappers to new sites in a cheaper way. However, in practice, active-learning methods not only constantly require human effort in building specialized annotation tools [24] but also need humans to label samples for each new site of interest. A recent work by Lockard et al. [25] attempted to use additional knowledge bases as distant supervision to automatically label some samples in the target websites and then learn a machine learning model on such noisy-labeled data. A large and comprehensive knowledge base is not always accessible and available for every domain. Consequently, their methods do not apply for emerging domains without first requiring the human effort of building a knowledge base. To address the problem in a more domain-general setting, we do not impose any human prior knowledge on the values, and focus on a purely unsupervised model adaptation learning scenario.

Transferable Extraction Models. Hao et al. [14] proposed a method that was based on visual distance features on a rendered web page. This achieved promising results on unseen websites without using any new human annotations. Following their work, there are a series of rendering-based extraction models proposed for many different settings. However, these rendering-based methods need to download all the external files including CSS style files, javascripts and images such that they can render a page with browser engines to know page layouts [31]. In addition, they have to design heuristic algorithms for computing human-crafted distance metrics $[4,47]$. These drawbacks together make large-scale extraction less practical and efficient. Our method, FreEDOM, instead is totally based on the HTML content itself without any use of external resources or page renders. When using two or more seed websites to train, FrEEDOM outperforms even the approach with expensive human-crafted features on visually-rendered pages.

Neural Architectures for Information Extraction. Another advantage of our method is that it can work well without any human-crafted features or patterns. We adopt state-of-the-art sequence modeling techniques [19,43] from IE and NLP to build our char-based word representations, XPath LSTMs, and position embeddings. Our pair-level network is inspired by the relational reasoning networks [36], which learns to induce relational features between semantic units (a DOM node in our case). A similar idea of encoding word-pairs as vectors also show promising results for reasoning about cross-sentence relations in natural language processing [15]. These together help us eliminate the need for human effort in both designing features and metric algorithms. The learned website-invariant neural features further make our model more transferable. Recent advances in natural language processing also show an emerging interest in learning powerful deep neural features for richly formatted texts and semi-structured data [16, 34]. Our work agrees with the findings in Fonduer [42]: neural networks can effectively replace hand-crafted features for extracting data in richly formatted documents. To the best of our knowledge, we are among the first approach using neural networks for learning to represent DOM nodes in web pages to solve structured prediction.

\section{CONCLUSIONS AND FUTURE WORK}

In this paper, we propose a neural architecture for extracting structured data from web documents. It uses training data from only a few seed sites but generalizes well to other unseen websites in the same vertical. We show that our approach, FreeDOM, beats the previous state-of-the-art performance on a large-scale public dataset consisting of 8 different verticals ( 80 websites in total) by nearly $3.7 \mathrm{~F} 1$ points. In particular, it does so without using any expensive rendering-based visual features. 
We believe that this work opens up multiple avenues for future research in web data extraction. What structured prediction techniques might work better at incorporating information from farther away and work well on large DOM trees with sparse labels? An even more interesting question is if we can transfer information across verticals? That is, if we are able to do well on one vertical, can we leverage that information somehow to train a model for the next vertical [26]? Will there be a large pre-trained neural encoding model for HTML documents, just like BERT [7] for plain texts?

\section{REFERENCES}

[1] Mohd Amir Bin Mohd Azir and Kamsuriah Ahmad. Wrapper approaches for web data extraction : A review. pages 1-6, 2017.

[2] Andrew Carlson and Charles Schafer. Bootstrapping information extraction from semi-structured web pages. In foint European Conference on Machine Learning and Knowledge Discovery in Databases, pages 195-210. Springer, 2008.

[3] Chia-Hui Chang, Mohammed O. Kayed, Moheb R. Girgis, and Khaled F. Shaalan. A survey of web information extraction systems. IEEE Transactions on Knowledge and Data Engineering, 18:1411-1428, 2006

[4] Joseph Paul Cohen, Wei Ding, and Abraham Bagherjeiran. Semi-supervised web wrapper repair via recursive tree matching. ArXiv, abs/1505.01303, 2015.

[5] Valter Crescenzi, Giansalvatore Mecca, and Paolo Merialdo. Roadrunner: Towards automatic data extraction from large web sites. In $V L D B, 2001$.

[6] Wanyun Cui, Yanghua Xiao, Haixun Wang, Yangqiu Song, Seung won Hwang, and Wei Yang Wang. Kbqa: Learning question answering over qa corpora and knowledge bases. PVLDB, 10:565-576, 2017.

[7] Jacob Devlin, Ming-Wei Chang, Kenton Lee, and Kristina Toutanova. Bert: Pretraining of deep bidirectional transformers for language understanding. In NAACL-HLT, 2019.

[8] Xin Dong, Evgeniy Gabrilovich, Geremy Heitz, Wilko Horn, Ni Lao, Kevin Murphy, Thomas Strohmann, Shaohua Sun, and Wei Zhang. Knowledge vault: a web-scale approach to probabilistic knowledge fusion. In KDD, 2014.

[9] Xin Dong, Evgeniy Gabrilovich, Geremy Heitz, Wilko Horn, Kevin Murphy, Shaohua Sun, and Wei Zhang. From data fusion to knowledge fusion. PVLDB, 7: 881-892, 2014.

[10] Emilio Ferrara, Pasquale De Meo, Giacomo Fiumara, and Robert Baumgartner Web data extraction, applications and techniques: A survey. Knowledge-based systems, 70:301-323, 2014

[11] Anna Lisa Gentile, Ziqi Zhang, and Fabio Ciravegna. Web scale information extraction with lodie. In AAAI Fall Symposia, 2013.

[12] Pankaj Gulhane, Rajeev Rastogi, Srinivasan H. Sengamedu, and Ashwin Tengli. Exploiting content redundancy for web information extraction. In WWW, 2010

[13] Pankaj Gulhane, Amit Madaan, Rupesh R. Mehta, Jeyashankher Ramamirtham, Rajeev Rastogi, Sandeepkumar Satpal, Srinivasan H. Sengamedu, Ashwin Tengli, and Charu Tiwari. Web-scale information extraction with vertex. 2011 IEEE 27th International Conference on Data Engineering, pages 1209-1220, 2011.

[14] Qiang Hao, Rui Cai, Yanwei Pang, and Lei Zhang. From one tree to a forest: a unified solution for structured web data extraction. In SIGIR, 2011

[15] Mandar Joshi, Eunsol Choi, Omer Levy, Daniel Weld, and Luke Zettlemoyer pair2vec: Compositional word-pair embeddings for cross-sentence inference. In Proceedings of NAACL-HLT, 2019.

[16] Furkan Kocayusufoglu, Ying Sheng, Nguyen Vo, James Wendt, Qi Zhao, Sandeep Tata, and Marc Najork. Riser: Learning better representations for richly structured emails. In The World Wide Web Conference, pages 886-895. ACM, 2019.

[17] Nicholas Kushmerick, Daniel S. Weld, and Robert B. Doorenbos. Wrapper induction for information extraction. In IFCAI, 1997

[18] John D. Lafferty, Andrew McCallum, and Fernando Pereira. Conditional random fields: Probabilistic models for segmenting and labeling sequence data. In ICML, 2001.

[19] Guillaume Lample, Miguel Ballesteros, Sandeep Subramanian, Kazuya Kawakami, and Chris Dyer. Neural architectures for named entity recognition. In HLTNAACL, 2016.

[20] Xiang Li, Yanxu Zhu, Gang Yin, Tao Wang, and Huaimin Wang. Exploiting attribute redundancy in extracting open source forge websites. 2012 International Conference on Cyber-Enabled Distributed Computing and Knowledge Discovery, pages 13-20, 2012.

[21] Bill Y. Lin, Frank F. Xu, Zhiyi Luo, and Kenny Q. Zhu. Multi-channel bilstm-crf model for emerging named entity recognition in social media. In NUT@EMNLP, 2017.

[22] Bill Yuchen Lin and Wei Lu. Neural adaptation layers for cross-domain named entity recognition. In $E M N L P, 2018$

[23] Bill Yuchen Lin, Xinyue Chen, Jamin Chen, and Xiang Ren. KagNet: Knowledgeaware graph networks for commonsense reasoning. In Proceedings of the 2019 Conference on Empirical Methods in Natural Language Processing and the 9th
International foint Conference on Natural Language Processing, 2019.

[24] Bill Yuchen Lin, Dong-Ho Lee, Frank F. Xu, Ouyu Lan, and Xiang Ren. AlpacaTag: An active learning-based crowd annotation framework for sequence tagging. In Proceedings of the 57th Annual Meeting of the Association for Computational Linguistics: System Demonstrations, 2019.

[25] Colin Lockard, Xin Luna Dong, Prashant Shiralkar, and Arash Einolghozati. CERES: distantly supervised relation extraction from the semi-structured web. PVLDB, 11(10):1084-1096, 2018. doi: 10.14778/3231751.3231758.

[26] Colin Lockard, Prashant Shiralkar, Xin Dong, and Hannaneh Hajishirzi. Zeroshotceres: Zero-shot relation extraction from semi-structured webpages. In Proceedings of ACL, 2020.

[27] Zhiyi Luo, Shanshan Huang, Frank F. Xu, Bill Yuchen Lin, Hanyuan Shi, and Kenny Q. Zhu. Extra: Extracting prominent review aspects from customer feedback. In EMNLP, 2018.

[28] Weizhi Ma, Min Zhang, Yue Cao, Woojeong Jin, Chenyang Wang, Yiqun Liu, Shaoping $\mathrm{Ma}$, and Xiang Ren. Jointly learning explainable rules for recommendation with knowledge graph. In $W W W, 2019$.

[29] Xuezhe Ma and Eduard Hovy. End-to-end sequence labeling via bi-directional lstm-cnns-crf. arXiv preprint arXiv:1603.01354, 2016.

[30] Sergey Melnik, Andrey Gubarev, Jing Jing Long, Geoffrey Romer, Shiva Shivakumar, Matt Tolton, and Theo Vassilakis. Dremel: interactive analysis of web-scale datasets. Commun. ACM, 54:114-123, 2011.

[31] Adi Omari, Benny Kimelfeld, Eran Yahav, and Sharon Shoham. Lossless separation of web pages into layout code and data. In Proceedings of the 22nd ACM SIGKDD international conference on knowledge discovery and data mining, pages 1805-1814, 2016.

[32] Jeffrey Pennington, Richard Socher, and Christopher D. Manning. Glove: Global vectors for word representation. In Empirical Methods in Natural Language Processing (EMNLP), pages 1532-1543, 2014.

[33] Dustin Arendt Prasha Shresha, Suraj Maharjan and Svitlana Volkova. Forecasting social interactions from dynamic graphs: A case study of twitter, github, and youtube. In Proceedings of the 15th International Workshop on Mining and Learning with Graphs (MLG), 2019.

[34] Yujie Qian, Enrico Santus, Zhijing Jin, Jiang Guo, and Regina Barzilay. Graphie: A graph-based framework for information extraction. In NAACL-HLT, 2018.

[35] Xiang Ren, Wenqi He, Meng Qu, Lifu Huang, Heng Ji, and Jiawei Han. Afet: Automatic fine-grained entity typing by hierarchical partial-label embedding. In EMNLP, 2016.

[36] Adam Santoro, David Raposo, David G. T. Barrett, Mateusz Malinowski, Razvan Pascanu, Peter W. Battaglia, and Timothy P. Lillicrap. A simple neural network module for relational reasoning. In NIPS, 2017.

[37] Andreas Schulz, Jörg Lässig, and Martin Gaedke. Practical web data extraction: are we there yet?-a short survey. In 2016 IEEE/WIC/ACM International Conference on Web Intelligence (WI), pages 562-567. IEEE, 2016.

[38] Hassan A. Sleiman and Rafael Corchuelo. A survey on region extractors from web documents. IEEE Transactions on Knowledge and Data Engineering, 25:1960-1981, 2013.

[39] Alon Talmor and Jonathan Berant. The web as a knowledge-base for answering complex questions. In Proceedings of the 2018 Conference of the North American Chapter of the Association for Computational Linguistics: Human Language Technologies, Volume 1 (Long Papers), pages 641-651, New Orleans, Louisiana, June 2018. Association for Computational Linguistics. doi: 10.18653/v1/N18-1059.

[40] Rakshit Trivedi, Hanjun Dai, Yichen Wang, and Le Song. Know-evolve: Deep temporal reasoning for dynamic knowledge graphs. In ICML, 2017.

[41] Hongwei Wang, Fuzheng Zhang, Miao Zhao, Wenjie Li, Xing Xie, and Minyi Guo. Multi-task feature learning for knowledge graph enhanced recommendation. In $W W W, 2019$.

[42] Sen Wu, Luke Hsiao, Xiao Cheng, Braden Hancock, Theodoros Rekatsinas, Philip Levis, and Christopher Ré. Fonduer: Knowledge base construction from richly formatted data. Proceedings of the 2018 International Conference on Management of Data, 2018.

[43] Zichao Yang, Diyi Yang, Chris Dyer, Xiaodong He, Alexander J. Smola, and Eduard H. Hovy. Hierarchical attention networks for document classification. In HLT-NAACL, 2016

[44] Daojian Zeng, Kang Liu, Siwei Lai, Guangyou Zhou, and Jun Zhao. Relation classification via convolutional deep neural network. In COLING, 2014.

[45] Yanhong Zhai and Bing Liu. Web data extraction based on partial tree alignment. In $W W W, 2005$.

[46] Wenxuan Zhou, Hongtao Lin, Bill Yuchen Lin, Ziqi Wang, Junyi Du, Leonardo Neves, and Xiang Ren. Nero: A neural rule grounding framework for labelefficient relation extraction. In Proceedings of The Web Conference 2020, WWW '20, page 2166-2176, New York, NY, USA, 2020. Association for Computing Machinery. ISBN 9781450370233. doi: 10.1145/3366423.3380282.

[47] Jun Zhu, Zaiqing Nie, Ji-Rong Wen, Bo Zhang, and Wei-Ying Ma. 2d conditional random fields for web information extraction. In ICML, 2005. 


\section{A APPENDIX}

Here we introduce more implementation details and some additional analysis of the proposed method in order to make our work more reproducible.

\section{A.1 Implementation Details.}

Filtering Fixed DOM Nodes. As we know, detail pages in a website usually share a lot of common boilerplate (e.g. navigation texts, headers, footers, etc) where the textual nodes do not convey variable values of our interested fields $[12,20]$. One can quickly eliminate them from consideration to reduce the number of nodes we are labeling in the model. We propose a simple heuristic to utilize sitelevel information to filter nodes that are constant values in all pages of a website. Specifically, we first collect all possible XPaths (i.e. node identifiers) of textual nodes in the website and then keep the number of different texts inside each node.

Then, we rank all nodes by these numbers, and take top-k of the XPaths with more than two different values as the variable nodes (we set $\mathrm{k}$ as 500 in the experiments). This step can remove most of the page-invariant textual nodes which are irrelevant information for data extraction such as copyright information, website names, and etc. The basic assumption behind this filtering is that we believe value nodes are more likely to have more different texts inside different pages and at least show two different values. In this way, we can significantly reduce our training and inference time without losing performance.

\section{A.2 Hyper-parameters}

We show our hyper-parameters and detailed model architectures.

The Node Encoder Module. First, the character-embedding dimension $\operatorname{dim}_{c}=100$, the word embeddings dimension $\operatorname{dim}_{w}=100$, the discrete feature embeddings is $\operatorname{dim}_{d}$ is 20 for the leaf type tag feature and 30 for the node value-checker features. For the CNN network (i.e. the char-level word encoder), we use 50 filters and 3 as kernel size. We set the LSTM of node text and preceding texts are both 100 dimensions. Therefore, we learned $300 \mathrm{~d}$ node vectors at the end of this stage. We train the node encoder with epoch number as 10 and a batch size of 16 . For our ablation studies with NL-based sequence tagging tasks, we use a 100d LSTM or a 100 -fileter CNNs for the node tagger.

The Pair-level Relation Network Module. The XPath and position embedding vectors are having 30 as dimension, and the XPath LSTM has a 100-dimension hidden size. The MLP for the pair classifier is also a $100 \mathrm{~d}$ dense layer. We train the relation network with epoch number as 10 and a batch size of 32 . We empirically set the threshold of $N$ to be 1 and the $m$ to be 10 for pair construction and label aggregation.

Shared training parameters. We use dropout layers at the end of both stages with a rate as 0.3 for avoid over-fitting. For the optimizer, we use Adam for both stages, with the learning_rate $=0.001$, beta_1 $=0.9$, beta_2 $=0.999$, and epsilon $=1 \mathrm{e}-07$.

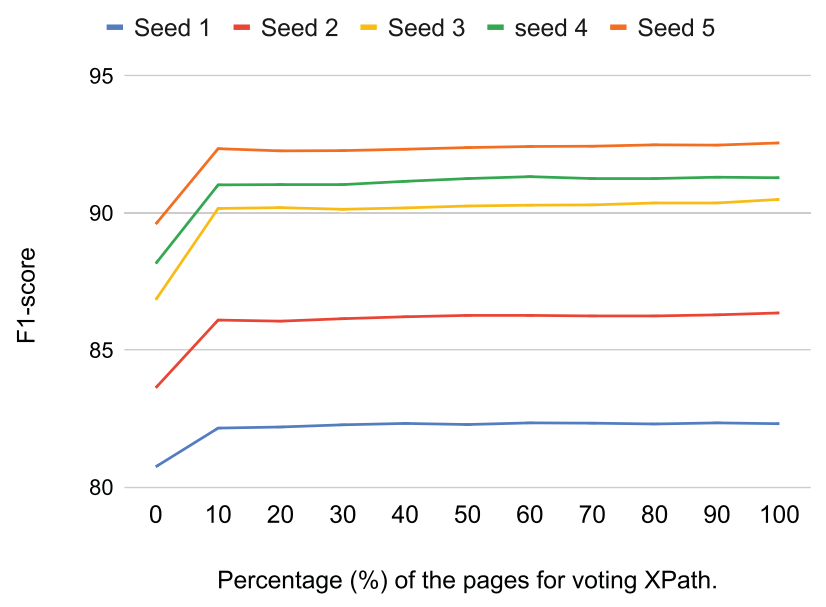

Figure 8: The curve of F1-score v.s. the percentage of the pages used for site-level voting, showing that even with streaming test data our methods still can achieve comparable performance under low-resource settings.

\section{A.3 More Analysis of FreeDOM}

A.3.1 Effect of site-level voting.

More specifically, for each field $f_{k}$, we iterate all the perfections for $f_{k}$ in all the testing pages and then we rank the XPaths by their number of occurrence. Then, in the second processing of each page, we rank all the XPaths in this page and order them with the previous computed occurrence scores, take the highest node as $f_{k}$ as well if it is labeled as other labels. Fig. 8 shows the curve when we use different proportion $(0 \%, 10 \%, \ldots, 100 \%)$ of the pages for voting XPaths. The results with growing percentages used for majority voting can be a good estimation when the system is applied in online streaming scenarios where inputs are emerging unseen pages from different websites.

A.3.2 A closer look at a vertical. We use an experiment in the Book vertical using three seed websites $(k=3)$ and compare the results from the first stage model and the second stage model. Table 4 clearly shows the improvement for each case when using our proposed pair-level networks for encoding relational information. The overall performance over these ten permutations of seed websites has a significant increment $(88.90 \rightarrow 93.90)$. One of the largest improvements is from "barnes.+bdep.+bsam." to "abe." $(79.20 \rightarrow 98.60)$.

We analyze the distance patterns of the abe. pages and the three seed sites, and find that they share a similar inter-field patterns in (as our previous discussion about Figure 9). This suggests that the second-stage module indeed captures the relational patterns. 


\begin{tabular}{|c|c|c|c|c|c|c|c|c|c|c|c|}
\hline Seed $\backslash$ Target Site & abe. & amazon & barnes. & bdep. & bsam. & borders & buy & chris. & dee. & water. & AVG \\
\hline abe.+amazon+barnes. & N/A & N/A & $\mathrm{N} / \mathrm{A}$ & $99.00 \rightarrow 99.00$ & $99.67 \rightarrow 100.00$ & $100.00 \rightarrow 100.00$ & $66.67 \rightarrow 91.33$ & $96.67 \rightarrow 96.67$ & $100.00 \rightarrow 99.67$ & $98.67 \rightarrow 98.67$ & $94.38 \rightarrow 97.90$ \\
\hline amazon+barnes.+bdep. & $99.67 \rightarrow 99.67$ & $\mathrm{~N} / \mathrm{A}$ & N/A & $\mathrm{N} / \mathrm{A}$ & $70.67 \rightarrow 78.67$ & $99.67 \rightarrow 99.67$ & $69.33 \rightarrow 91.00$ & $97.33 \rightarrow 97.33$ & $100.00 \rightarrow 100.00$ & $99.00 \rightarrow 98.67$ & $90.81 \rightarrow 95.00$ \\
\hline barnes.+bdep.+bsam. & $78.80 \rightarrow 98.40$ & $79.50 \rightarrow 84.50$ & N/A & N/A & N/A & $99.80 \rightarrow 99.80$ & $82.20 \rightarrow 93.00$ & $74.80 \rightarrow 94.00$ & $98.00 \rightarrow 98.20$ & $99.40 \rightarrow 98.60$ & $87.50 \rightarrow 95.21$ \\
\hline bdep.+bsam.+borders & $\underline{79.20 \rightarrow 98.60}$ & $78.25 \rightarrow 80.25$ & $99.60 \rightarrow 99.40$ & N/A & N/A & N/A & $98.00 \rightarrow 95.00$ & $75.80 \rightarrow 94.80$ & $99.60 \rightarrow 99.40$ & $99.60 \rightarrow 99.00$ & $90.01 \rightarrow 95.21$ \\
\hline bsam.+borders+buy & $\overline{78.60 \rightarrow 98.00}$ & $58.00 \rightarrow 63.75$ & $99.60 \rightarrow 99.40$ & $79.60 \rightarrow 82.00$ & N/A & N/A & N/A & $64.00 \rightarrow 93.00$ & $92.00 \rightarrow 98.60$ & $99.00 \rightarrow 98.60$ & $81.54 \rightarrow 90.48$ \\
\hline borders+buy+chris. & $91.40 \rightarrow 92.00$ & $74.50 \rightarrow 95.00$ & $78.80 \rightarrow 94.00$ & $98.60 \rightarrow 97.40$ & $97.40 \rightarrow 97.00$ & N/A & N/A & N/A & $99.00 \rightarrow 98.60$ & $99.00 \rightarrow 98.80$ & $91.24 \rightarrow 96.11$ \\
\hline buy+chris.+dee. & $90.20 \rightarrow 90.20$ & $74.50 \rightarrow 74.50$ & $79.60 \rightarrow 82.60$ & $99.00 \rightarrow 98.20$ & $59.20 \rightarrow 81.40$ & $79.40 \rightarrow 99.00$ & N/A & N/A & $\mathrm{N} / \mathrm{A}$ & $99.60 \rightarrow 99.00$ & $83.07 \rightarrow 89.27$ \\
\hline chris.+dee.+water. & $97.40 \rightarrow 97.40$ & $69.25 \rightarrow 75.75$ & $74.20 \rightarrow 96.40$ & $96.60 \rightarrow 94.00$ & $50.20 \rightarrow 80.40$ & $99.40 \rightarrow 98.80$ & $77.60 \rightarrow 80.40$ & $\mathrm{~N} / \mathrm{A}$ & N/A & N/A & $80.66 \rightarrow 89.02$ \\
\hline dee.+water.+abe. & $\mathrm{N} / \mathrm{A}$ & $71.75 \rightarrow 77.50$ & $79.60 \rightarrow 99.40$ & $98.20 \rightarrow 87.80$ & $99.00 \rightarrow 99.00$ & $99.80 \rightarrow 99.80$ & $95.80 \rightarrow 90.40$ & $97.40 \rightarrow 97.60$ & N/A & N/A & $91.65 \rightarrow 93.07$ \\
\hline water.+abe.+amazon & N/A & N/A & $98.67 \rightarrow 99.00$ & $94.33 \rightarrow 95.00$ & $100.00 \rightarrow 100.00$ & $99.67 \rightarrow 99.67$ & $97.00 \rightarrow 93.67$ & $97.67 \rightarrow 97.67$ & $99.33 \rightarrow 99.33$ & N/A & $98.10 \rightarrow 97.76$ \\
\hline AVG & $87.90 \rightarrow 96.32$ & $72.25 \rightarrow 78.75$ & $87.15 \rightarrow 95.74$ & $95.05 \rightarrow 93.34$ & $82.31 \rightarrow 90.92$ & $96.82 \rightarrow 99.53$ & $83.80 \rightarrow 90.69$ & $86.24 \rightarrow 95.87$ & $98.28 \rightarrow 99.11$ & $99.18 \rightarrow 98.76$ & $88.90 \rightarrow 93.90$ \\
\hline
\end{tabular}

Table 4: A detailed case study in the Book vertical for comparing the first-stage and second-stage model prediction results when $k=3$. Each entry in the table shows the improvement from first-stage results to $(\rightarrow)$ second-stage ones.

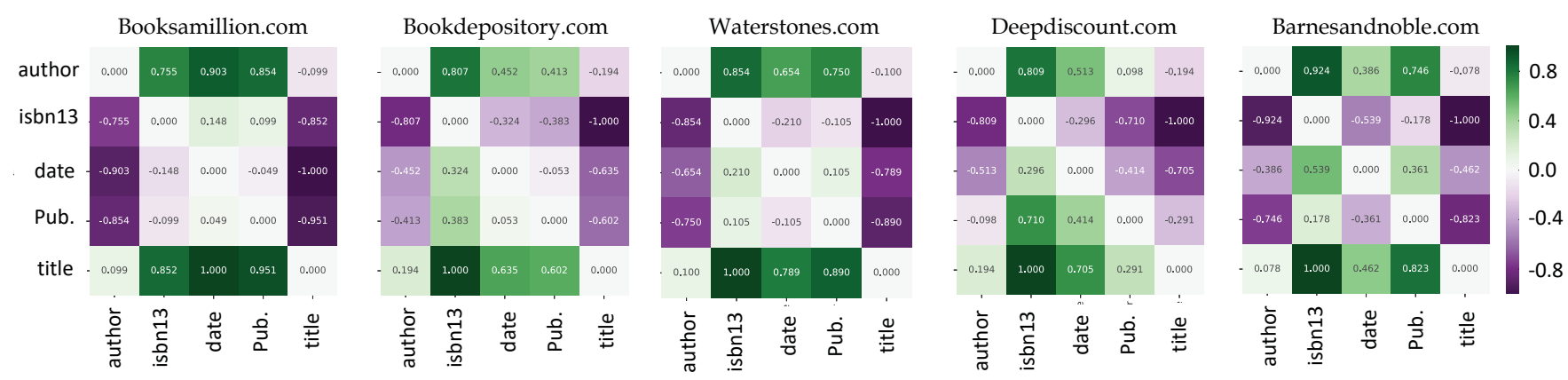

Figure 9: Patterns of distances between the value nodes of field-pairs in different websites of Book vertical. The distance values are site-level normalized with MinMaxScaler, thus ranging form -1 to +1 . Positive distances (in green) means the columnfield is behind row-field. Similarly, the negative distances (in purple) mean that the row-fields are behind column-fields. The distance values in the figure are the number of variable nodes are there between two fields which are further normalized by the total number of nodes. 